\title{
Glucose Metabolism as a Pre-clinical Biomarker for the Golden Retriever Model of Duchenne Muscular Dystrophy
}

\author{
Sarah Morar Schneider, ${ }^{1}$ Vidya Sridhar, ${ }^{2}$ Amanda K. Bettis, ${ }^{3}$ Heather Heath-Barnett, ${ }^{3}$ \\ Cynthia J. Balog-Alvarez, ${ }^{3}$ Lee-Jae Guo, ${ }^{2,3}$ Rachel Johnson, ${ }^{2}$ Scott Jaques, ${ }^{4}$ \\ Stanislav Vitha, ${ }^{5}$ Alan C. Glowcwski, ${ }^{2}$ Joe N. Kornegay, ${ }^{3}$ Peter P. Nghiem ${ }^{3}$ \\ ${ }^{1}$ Department of Veterinary Pathobiology, College of Veterinary Medicine and Biomedical Sciences, Texas A\&M University, College Station, \\ TX, 77843-4458, USA \\ ${ }^{2}$ Texas A\&M Institute for Preclinical Studies, College of Veterinary Medicine and Biomedical Sciences, Texas A\&M University, College \\ Station, TX, 77843-4458, USA \\ ${ }^{3}$ Department of Veterinary Integrative Biosciences, College of Veterinary Medicine and Biomedical Sciences, Texas A\&M University, 4458 \\ TAMU, College Station, TX, 77843-4458, USA \\ ${ }^{4}$ Texas A\&M Veterinary Diagnostic Laboratory, College of Veterinary Medicine and Biomedical Sciences, Texas A\&M University, College \\ Station, TX, 77843-4458, USA \\ ${ }^{5}$ Microscopy Imaging Center, College of Veterinary Medicine and Biomedical Sciences, Texas A\&M University, College Station, TX, 77843- \\ 4458, USA
}

\begin{abstract}
Purpose: Metabolic dysfunction in Duchenne muscular dystrophy (DMD) is characterized by reduced glycolytic and oxidative enzymes, decreased and abnormal mitochondria, decreased ATP, and increased oxidative stress. We analyzed glucose metabolism as a potential disease biomarker in the genetically homologous golden retriever muscular dystrophy (GRMD) dog with molecular, biochemical, and in vivo imaging.

Procedures: Pelvic limb skeletal muscle and left ventricle tissue from the heart were analyzed by mRNA profiling, qPCR, western blotting, and immunofluorescence microscopy for the primary glucose transporter (GLUT4). Physiologic glucose handling was measured by fasting glucose tolerance test (GTT), insulin levels, and skeletal and cardiac positron emission tomography/X-ray computed tomography (PET/CT) using the glucose analog 2-deoxy-2-[ $\left[{ }^{18} \mathrm{~F}\right]$ fluoro-D-glucose $\left.\left({ }^{18} \mathrm{~F}\right] \mathrm{FDG}\right)$.

Results: MRNA profiles showed decreased GLUT4 in the cranial sartorius (CS), vastus lateralis (VL), and long digital extensor (LDE) of GRMD vs. normal dogs. QPCR confirmed GLUT4 downregulation but increased hexokinase-1. GLUT4 protein levels were not different in the CS, $\mathrm{VL}$, or left ventricle but increased in the LDE of GRMD vs. normal. Microscopy revealed diffuse membrane expression of GLUT4 in GRMD skeletal but not cardiac muscle. GTT showed higher basal glucose and insulin in GRMD but rapid tissue glucose uptake at 5 min post-dextrose injection in GRMD vs. normal/carrier dogs. PET/ CT with $\left[{ }^{18} \mathrm{~F}\right] \mathrm{FDG}$ and simultaneous insulin stimulation showed a significant increase $(p=0.03)$ in mean standard uptake values (SUV) in
\end{abstract}

Electronic supplementary material The online version of this article (https:// doi.org/10.1007/s11307-018-1174-2) contains supplementary material, which is available to authorized users.

Correspondence to: Peter Nghiem; e-mail: pnghiem@cvm.tamu.edu 
GRMD skeletal muscle but not pelvic fat at 5 min post- $\left[{ }^{18} \mathrm{~F}\right] \mathrm{FDG}$ /insulin injection. Conversely, mean cardiac SUV was lower in GRMD than carrier/normal $(p<0.01)$.

Conclusions: Altered glucose metabolism in skeletal and cardiac muscle of GRMD dogs can be monitored with molecular, biochemical, and in vivo imaging studies and potentially utilized as a biomarker for disease progression and therapeutic response.

Key words: GLUT4, GRMD, DMD, Dog, Glucose, Insulin, Metabolism, PET/CT, Biomarkers, Dystrophin

\section{Introduction}

Duchenne muscular dystrophy (DMD) is a muscle wasting disease in boys due to loss of dystrophin [1, 2]. Membrane fragility causes myofiber degeneration, leading to loss of ambulation in adolescence and eventual death from cardiorespiratory complications [3]. In addition to, or as a consequence of, membrane fragility, dystrophic muscle is under increased metabolic stress due to decreased glycolytic/ oxidative enzyme expression. There are also lower mitochondrial numbers, including some with abnormal structure, decreased intracellular ATP, and increased reactive oxygen species [4-9]. These alterations in dystrophic muscle lead to a "metabolic crisis" and a reduced capacity to respond to metabolic demands such as muscle contraction.

The main glucose transporter in cardiac and skeletal muscle [10], glucose transporter-4 (GLUT4), is abnormally located in subcellular aggregates in DMD [11]. In contrast, GLUT4 was found in membrane preps of a subcellular fractionation study in the $\mathrm{mdx}$ mouse model for DMD, suggesting a compensatory mechanism $[11,12]$. GLUT4 translocation is controlled by both insulin and muscle contraction, which act through independent molecular pathways to increase cytoplasmic vesicle translocation to the cell membrane. GLUT4 transports back to the cytoplasm after insulin stimulation or contraction are withdrawn [13]. Since different vesicle pools of GLUT4 respond to insulin and contraction [14, 15], these two pathways may be cumulative. The membrane localization of GLUT4 in $\mathrm{mdx}$ mice may have functional significance, because dystrophic myofibers have an altered response to insulin $[11,14,16]$.

Dogs with golden retriever muscular dystrophy (GRMD) have absent dystrophin $[17,18]$ and a more severe phenotype compared with mdx mice, suggesting that pre-clinical studies in affected dogs may better mirror anticipated outcomes in DMD. Specifically, GRMD dogs show a similar metabolic crisis in dystrophic muscle, with reduced glycolytic enzymes, decreased and abnormal mitochondria, and dysregulated AMPK expression $[4,19]$. We hypothesized that GRMD myofibers would have altered GLUT4 localization (similar to DMD muscle) and subsequently altered (reduced) glucose metabolism. Based on our initial findings, GLUT4 was surprisingly localized to the sarcolemmal membrane, which led to rapid clearance of blood glucose and increased muscle uptake of a glucose analog. Our results suggest that glucose metabolism can be monitored and potentially utilized as a preclinical biomarker in GRMD dogs.

\section{Materials and Methods}

Materials and methods are provided in the electronic supplementary material (ESM).

\section{Results}

\section{MRNA Profiling and $q P C R$}

GLUT-4 expression from genome-wide mRNA profiles [20, 21] of GLUT4 mRNA in the CS, LDE, and VL muscles of GRMD was decreased at 4-9 weeks and 6 months compared with normal, with differences being most pronounced in the LDE (Fig. 1a). Specifically, GLUT4 mRNA expression was approximately $50 \%$ less in GRMD LDE compared with normal, while the decrease in the CS and VL was only $\sim 10$ $25 \%$. The LDE in normal dogs had higher basal GLUT4 expression compared with the CS and VL. QPCR for GLUT4 in the GRMD LDE at 6 months confirmed its downregulation by a fold change of -1.894 (standard error of $\pm 0.20028 ; p=.0245$ ) (Fig. 1b). Interestingly, in the same set of GRMD LDE muscle, Hexokinase-1 (HK1) was upregulated by a fold change of + 2.13 (standard error $\pm 0.88863 ; p=.046$ ) (Fig. 1b).

\section{Western Blots}

GLUT4 protein expression did not significantly differ between normal and GRMD CS, VL, or left ventricle muscles (Fig. 2). GLUT4 protein expression at 6 months was significantly increased $(p<0.05)$ in GRMD LDE muscle, being nearly twice that of normal dogs.

\section{Confocal Microscopy}

GLUT4 was diffusely localized to the myofiber membrane in all GRMD skeletal muscles evaluated (CS, LDE, VL, cranial tibial, and diaphragm; $p=.047$; Fig. $3 \mathrm{~b}, \mathrm{~d}$, and g), whereas normal muscle showed minimal membrane localization (Fig. 3a, c). Cytoplasmic GLUT4 aggregates were present in both GRMD and normal muscle (Fig. 3a-d). There 


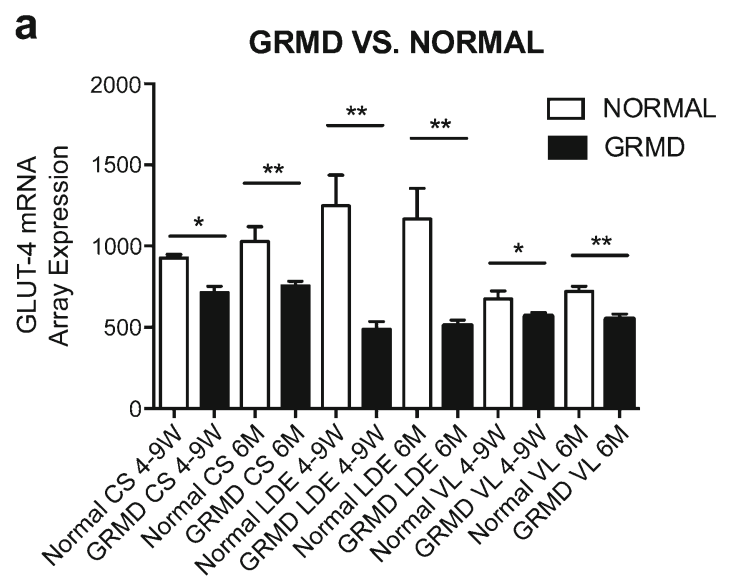

b

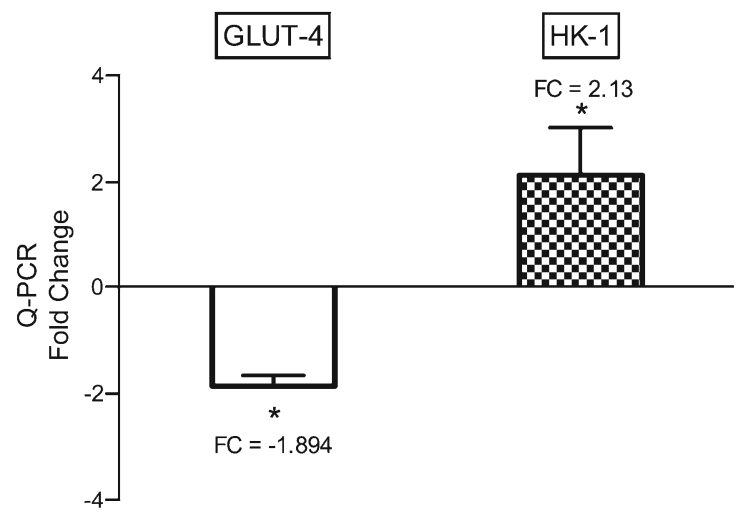

Fig. 1 . GLUT4 mRNA expression was reduced in GRMD vs. normal muscle. a GLUT4 mRNA expression was reduced in the $\mathrm{CS}$, VL, and most pronounced in the LDE (50 \% decrease) of GRMD (black bars) compared with normal (open bars) dogs at 49 weeks and 6 months. b QPCR for GLUT4 on GRMD LDE muscle at 6 months confirmed downregulation by a fold change of -1.894, while hexokinase 1 (HK-1) was upregulated by a fold change of $+2.13 .{ }^{*} p<0.05$; ${ }^{* *} p<0.01$.

was no difference in membrane expression between normal and GRMD left ventricle muscles from the heart (Fig. 3e, f).

\section{Intravenous Glucose Tolerance Test}

Based on the increased skeletal muscle membrane localization of GLUT4 demonstrated on confocal microscopy, we hypothesized that GRMD dogs would have more rapid clearance of plasma glucose and performed an intravenous glucose tolerance test (IV-GTT). For blood glucose (BG) values, both genotype $(p=0.002)$ and the interaction of time and genotype $(p=0.003)$ were significant. GRMD had higher basal BG levels compared with normal/carrier dogs (time 0; Fig. 4a). At 5 min post-dextrose injection, there was rapid tissue uptake of glucose from the blood in GRMD a

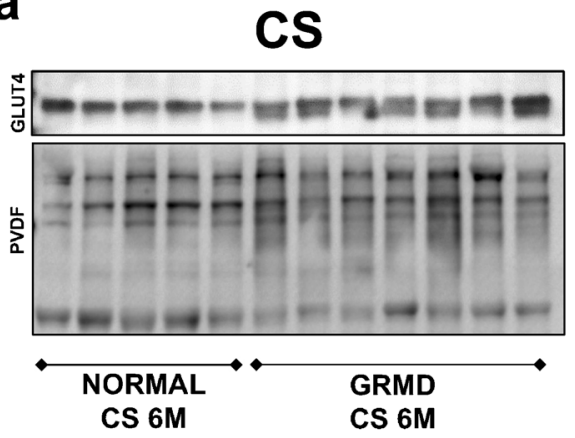

GLUT-4 PROTEIN

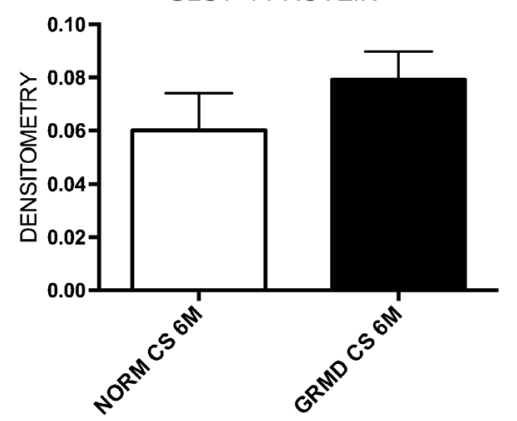

b

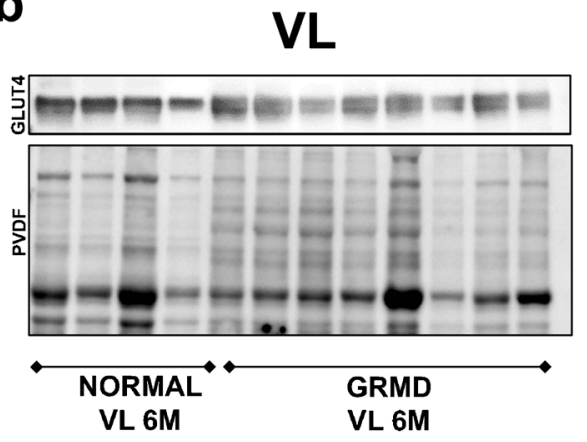

GLUT-4 PROTEIN

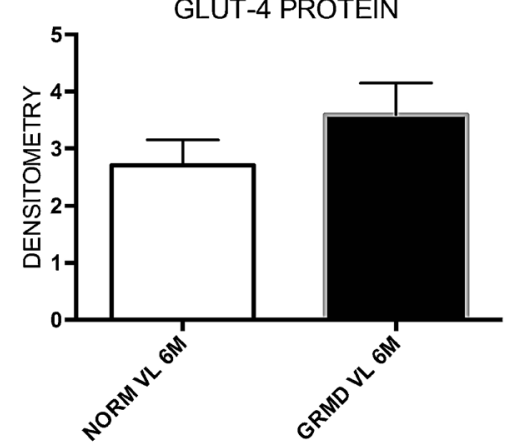

C
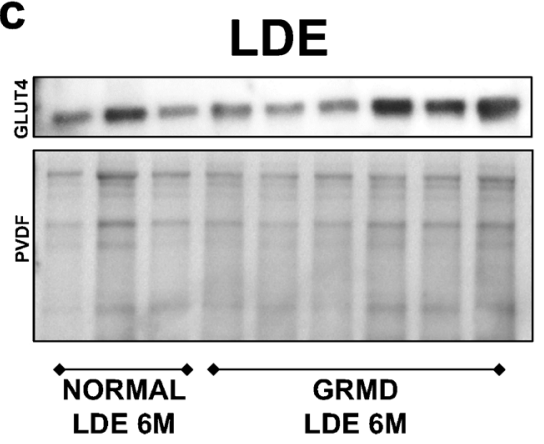

GLUT-4 PROTEIN

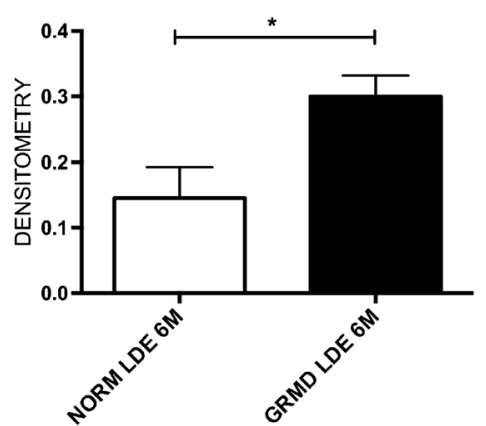

Fig. 2 GLUT4 protein in GRMD vs. normal muscles. GLUT4 expression was similar in a CS and b VL between GRMD (black bars) and normal (white) dogs, but increased in c GRMD LDE at 6 months. GLUT4 expression was normalized to total protein/ lane on the PVDF membrane. ${ }^{*} p<0.05$. 


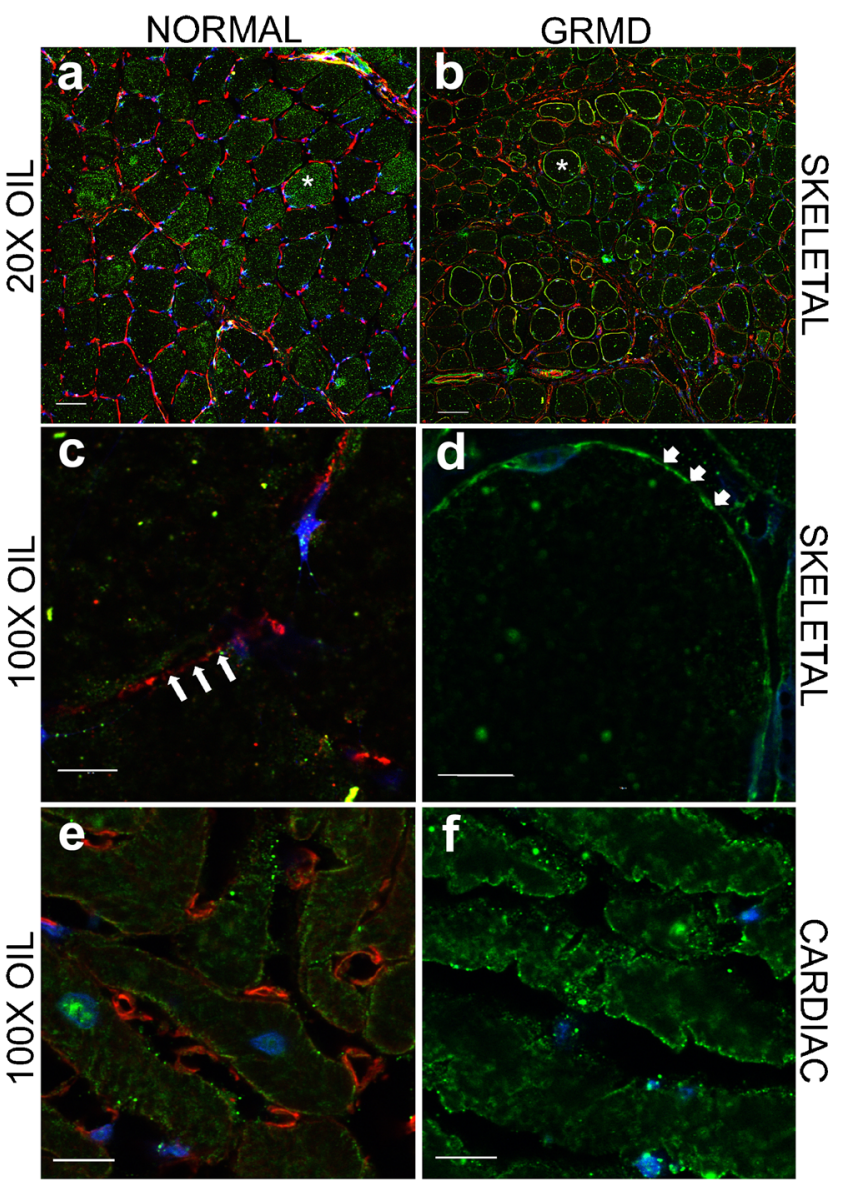

g

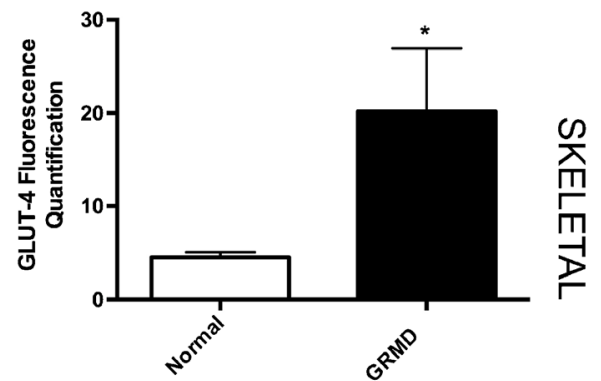

Fig. 3 Peri-membranous localization of GLUT4 in GRMD skeletal muscle. Confocal microscopy showed increased localization of GLUT4 (green) at the myofiber membrane (white asterisk and arrows) in b, d GRMD skeletal muscle (CS, LDE, VL, cranial tibial, diaphragm), compared with a, c normal. Mainly, cytoplasmic GLUT4 localization was observed in normal muscle (asterisk; a, c). Note that GRMD also demonstrated cytoplasmic GLUT4 aggregates. In cardiomyocytes, there was no statistical difference in GLUT-4 membrane expression between $\mathbf{e}$ normal and $\mathbf{f}$ GRMD. g Quantification showed a nearly fourfold increase of GLUT4 at the membrane in GRMD skeletal muscle $(p=$ 0.047 ); $\mathbf{a}, \mathbf{b} \times 20$ objective (oil immersion); $\mathbf{c}-\mathbf{f} \times 100$ objective (oil immersion). Nuclei, blue; spectrin, red. $N=5-6$ per group for skeletal muscle; $N=3$ per group for cardiac muscle.

(mean $507 \mathrm{mg} / \mathrm{dl}$ ) compared with carriers (mean $644 \mathrm{mg} / \mathrm{dl}$; $p<0.05$ ) and normal dogs (mean $597 \mathrm{mg} / \mathrm{dl} ; p<0.05$ ) (Fig. 4a). Normal and carrier BG values were not significantly different.

\section{Insulin Curve}

With regard to the IV-GTT, insulin values were also significantly affected by genotype $(p=0.007)$, although the interaction with time was not significant. There was a $45 \%$ increase in basal (time 0 ; pre-dextrose injection) insulin levels in GRMD (mean $31.26 \mu \mathrm{IU} / \mathrm{ml} ; p<0.05$ ) compared with combined normal/carrier groups (mean $21.61 \mu \mathrm{IU} / \mathrm{ml}$; $p<0.05$ ) (Fig. 4b). The GRMD insulin levels peaked at the 5-min time point, while both carrier and normal dogs did not peak until 15 min (Fig. 4b).

\section{PET/CT: Skeletal Muscle}

Based on the increased GLUT-4 membrane localization and initial rapid uptake of $\mathrm{BG}$, we hypothesized that in vivo imaging with positron emission tomograhy (PET)/X-ray computed tomography (CT) would be able to detect more rapid glucose uptake in GRMD skeletal muscle compared with normal/carrier dogs. With administration of 2-deoxy-2$\left[{ }^{18} \mathrm{~F}\right]$ fluoro-D-glucose ([ $\left.\left.{ }^{18} \mathrm{~F}\right] \mathrm{FDG}\right)$, a glucose analog, and coadministraion of insulin, genotype significantly increased mean skeletal muscle SUV at 5 min post-injection scan ( $p=$ 0.03 ) (Fig. 5a, b); this difference was lost at the $1-\mathrm{h}$ time point. The mean SUV was significantly different among the $\mathrm{CS}$, VL, and rectus femoris muscles at both time points $(p<0.01)$, regardless of genotype. Max SUV was not significantly different between muscles, genotype, or scan time.

To normalize for limb bloodflow, we assessed SUV in a nearby tissue (pelvic fat) that should not be influenced by disease status. Mean SUV in a 1-cm ROI of pelvic fat showed no significant differences between the groups at either time point; however, $\max$ SUV was significantly higher in normal dog pelvic fat $(p=0.03)$. There was no significant differences in systolic, diastolic, and mean blood pressure between groups at the time points before, just after, and $1 \mathrm{~h}$ after $\left[{ }^{18} \mathrm{~F}\right]$ FDG administration (Suppl. Table 1).

We hypothesized that severely affected muscles with heterogeneous muscle composition due to segmental necrosis, including areas of fibrosis, inflammation, degeneration, and myofiber regeneration would have more heterogeneous $\left[{ }^{18} \mathrm{~F}\right]$ FDG uptake. Using $\mathrm{CoV}$ (SD SUV/mean SUV) as a marker for heterogeneous uptake, values were significantly higher in GRMD VL muscle $(\mathrm{CoV}=0.204 ; p=0.02)$ vs. normal $\operatorname{dogs}(\mathrm{CoV}=0.174)$ at the $1-\mathrm{h}$ scan (Fig. 5c). Average $\mathrm{CoV}$ for carriers was midway between the GRMD and normal averages $(\mathrm{CoV}=0.181 ; p=0.29)$, although significance was not reached. The $\mathrm{CoV}$ of the hypertrophied CS muscle, which is relatively spared with minimal pathology (inflammation, degeneration) [20], did not differ between GRMD and normal/carrier dogs. 
a

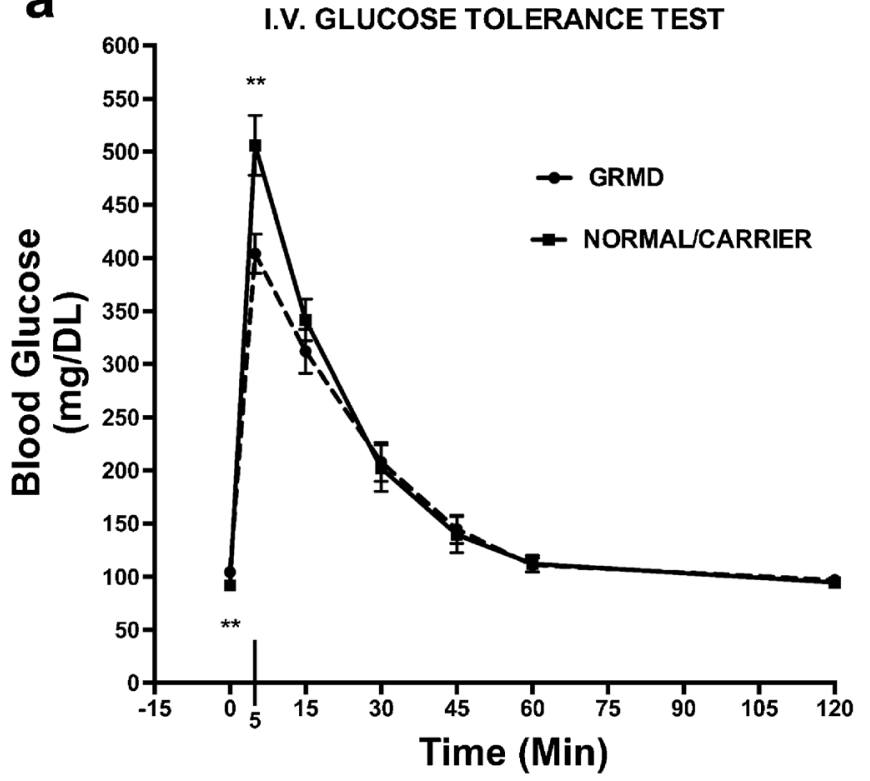

b

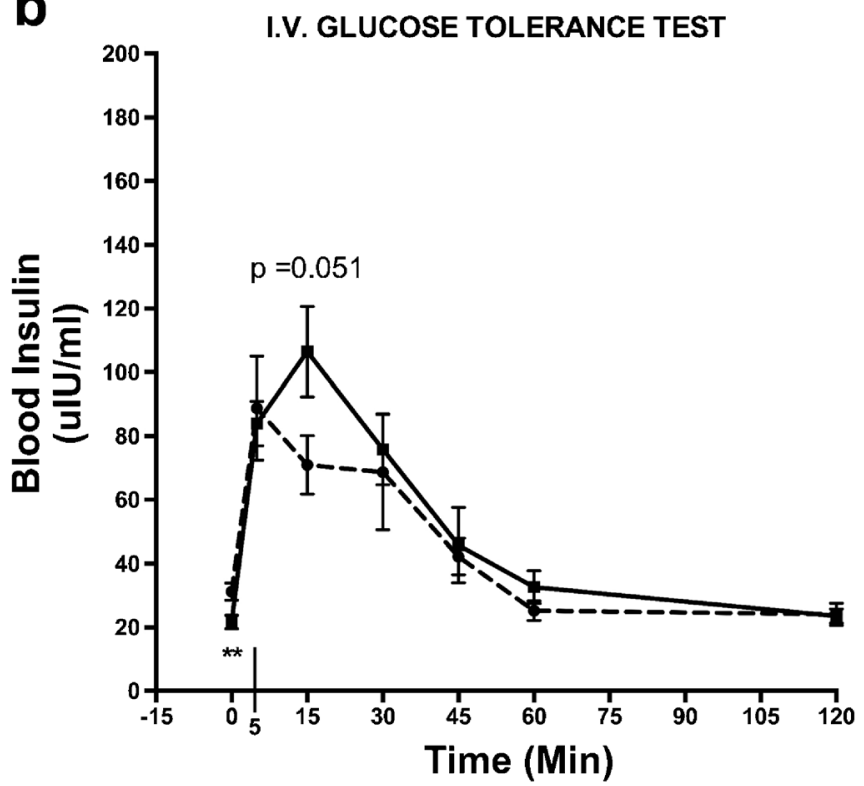

Fig. 4 Rapid dextrose uptake in GRMD with an intravenous glucose tolerance test. At early time points, $\mathbf{a}$ glucose and $\mathbf{b}$ insulin curves were significantly different between GRMD and carrier/normal dogs. Resting (basal, time $=0$ ) blood glucose was significantly higher in GRMD (104.5 mg/dl \pm 7.3$)$ compared with normal/carrier dogs (92.0 mg/dl \pm 8.9$)$. Dextrose uptake was rapid in GRMD dogs compared with normal at 5 min post-injection. Glucose levels were similar between genotypes at later time points. Resting (basal) insulin levels were significantly higher in GRMD dogs $(31.26 \mu \mathrm{lU} / \mathrm{ml} \pm 6.1 ; 35 \%$ increase) vs. normal and carrier dogs $(21.61 \mu \mathrm{lU} / \mathrm{ml} \pm 7.27)$ and GRMD insulin peaked at the 5-min time point compared with $15 \mathrm{~min}$ in carrier/normal dogs. Insulin levels were similar between genotypes at later time points. ${ }^{* *} p<0.01$.

\section{PET/CT: Cardiac Muscle}

Based on preferential involvement of the posterobasal heart region in DMD histopathologically and in previous imaging studies [22-24], we hypothesized that $\left[{ }^{18} \mathrm{~F}\right] \mathrm{FDG}$ uptake would be increased in these areas in GRMD. At the 5-min post- $\left[{ }^{18} \mathrm{~F}\right] \mathrm{FDG}$ /insulin scan, both mean $(2.7$ vs. $4.8 ; p=$ $0.007)$ and $\max (4.5 v s .7 .1 ; p=0.04)$ SUV were suprisingly lower in GRMD vs. carrier and normal hearts (Fig. 5d-h). At 1 -h post- $\left[{ }^{18} \mathrm{~F}\right] \mathrm{FDG}$ administration, mean SUV (2.8 vs. 4.7; $p=0.02$ ) was still significantly lower in GRMD, though the difference in max SUV (4.9 vs. 7.8; $p=0.06$ ) was no longer significant. Mean and max SUV did not differ among the standard $16 \mathrm{LV}$ segments or with $\mathrm{CoV}$ in the groups $(p>0.3)$.

\section{Discussion}

In addition to the mechanical fragility of the sarcolemma, dystrophin deficiency is characterized by metabolic dysregulation, manifesting as reduced glycolytic enzymes, mitochondrial structural and functional abnormalities, and altered glucose uptake and response to insulin [4, 8, 11, 19, 25-28]. Reduced muscle mass and increased fat are risk factors and could contribute to altered insulin sensitivity, independent of dystrophin. However, the presence of hyperglycemia and hypoglycemia in boys with normal or low body mass index [11] suggests that the dystrophy state, and not just body composition, alters insulin sensitivity in DMD. Cumulatively, these changes lead to a "metabolic crisis" in dystrophin deficient myofibers, which could result in greater susceptibility to ischemia, metabolic stress, and reduced regenerative capacity.

Glucose transport into muscle cells in response to insulin or contraction [29] occurs primarily through translocation of GLUT4 from the cytoplasm to the sarcolemma/T-tubules [10]. As such, alterations in GLUT4 levels or trafficking within the cell significantly impact overall glucose metabolism. Changes in GLUT4 localization has been noted in both DMD and mdx muscles, but it is unclear if this altered trafficking is a direct result of dystrophin loss or a secondary response to metabolic stress. We hypothesized that GLUT4 alterations in GRMD would be similar to DMD muscle [11], contributing to the metabolic dysregulation in dystrophic muscle.

We queried microarray profiles and found reduced GLUT4 mRNA in dystrophin-deficient muscles. However, our follow-up studies showed varying protein levels, being similar to normal dogs in GRMD CS and VL muscles, but increased in GRMD LDE. The protein results in the CS and VL are in keeping with unchanged levels of GLUT4 protein expression in type II diabetes, though mRNA is also unchanged in that disease [30, 31]. On the other hand, increased levels in GRMD LDE were consistent with elevated values in hind limb muscles of the mdx mouse, regardless of age [12]. Interestingly, GRMD LDE had the 

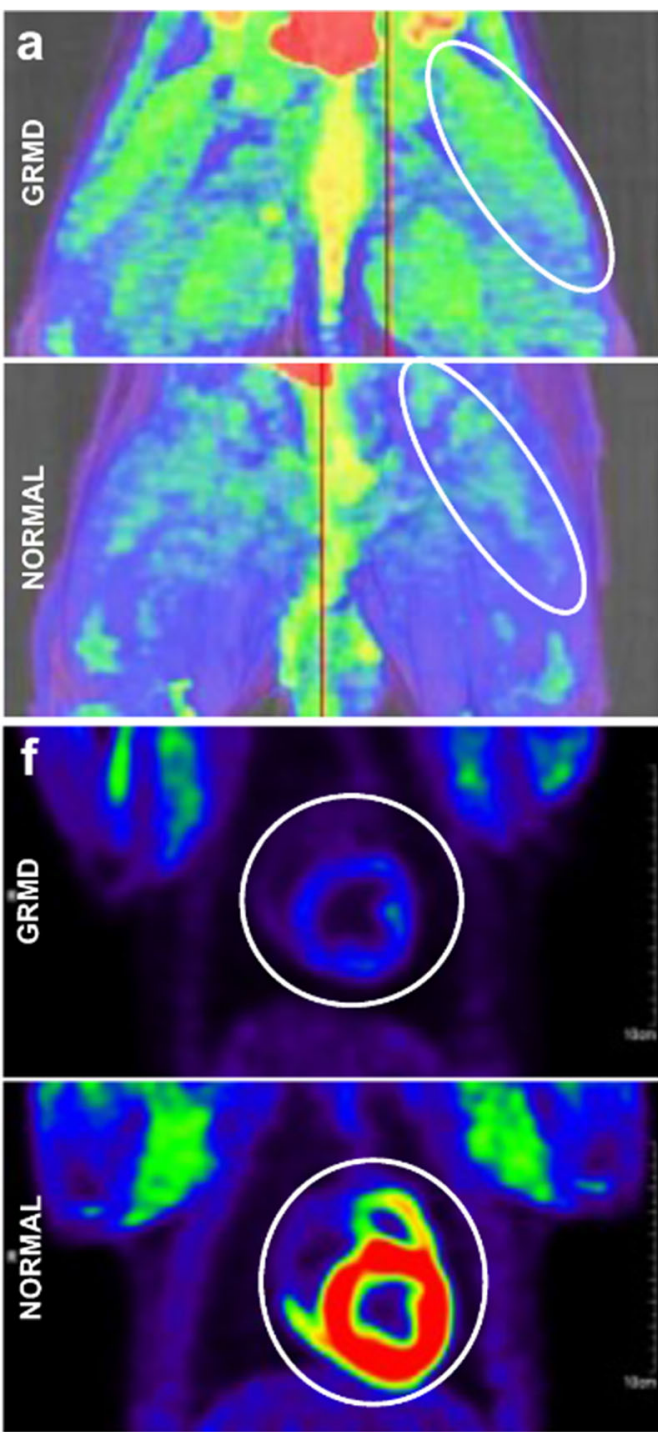
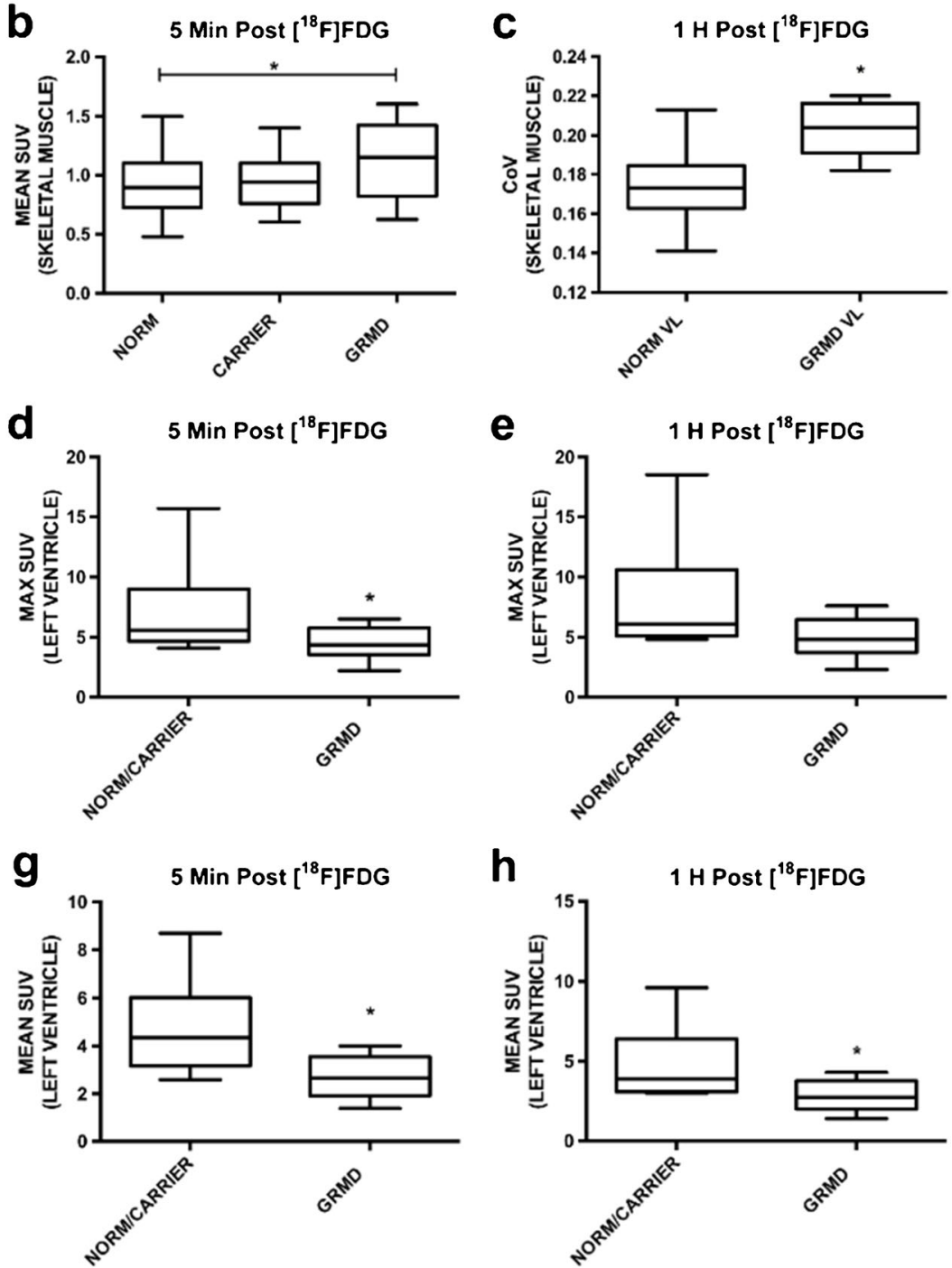

Fig. 5 Differential $\left[{ }^{18} \mathrm{~F}\right]$ FDG uptake with PET/CT in GRMD skeletal and cardiac muscle. a GRMD VL, rectus femoris, and CS (top, white oval) had higher SUV than normal littermates (bottom; white oval). b Skeletal muscle had increased mean SUV at 5 min post-[ $\left.{ }^{18} \mathrm{~F}\right] \mathrm{FDG} / \mathrm{insulin}$ in GRMD compared with normal. c GRMD skeletal muscle had significantly higher CoV at 1 h. In cardiac muscle, $\mathbf{d}$ SUVmax and $\mathbf{g}$ SUVmean were lower in the GRMD $\mathbf{f}$ left ventricle (top) compared with normal (bottom) and carrier at $5 \mathrm{~min}$ post-[ $\left.{ }^{18} \mathrm{~F}\right] \mathrm{FDG} /$ insulin (white circle outlining the heart). At $1 \mathbf{h}$, e SUVmax trended and $\mathbf{h}$ SUVmean were significantly lower in GRMD compared with normal left ventricle. ${ }^{*} p<0.05$.

most profound changes in GLUT4 mRNA and protein, suggesting a negative feedback loop may be involved between mRNA and protein. Moreover, these molecular changes in GRMD LDE may be due to the severe dystrophic phenotype and significant degeneration/regeneration observed in this muscle (LDE is atrophied/wasted to half the size of normal) [32]. With regard to the left ventricle of the heart, there was a trend for increased GLUT-4 in GRMD $(p=0.06)$. Perhaps with the availability of more samples, statistical significance could be achieved.

While overall GLUT4 protein levels in the tissues were not increased in most GRMD muscles evaluated, immunofluorescence microscopy surprisingly revealed increased GLUT4 localization at the myofiber cell membrane in all
GRMD skeletal muscle evaluated. This membrane localization could represent a dysregulation in cellular trafficking or a physiological response to increased metabolic demand for glucose (due to the metabolic crisis). On that note, GLUT4 translocation to the muscle membrane in this model may represent a sort of "priming" to allow for rapid extracellular glucose uptake. Interestingly, normal and GRMD muscles had similar amounts of cytoplasmic GLUT4 aggregates, representing a potential therapeutic reservoir for the latter. With regard to cardiomyocytes, we did not detect a statistical difference in GLUT4 membrane expression between normal and GRMD left ventricle samples. Lesions and associated clinical disease occur much earlier in skeletal vs. cardiac muscle in DMD and GRMD, raising questions of factors that 
could either accelerate skeletal muscle or delay cardiac involvement. These alterations in skeletal $v s$. cardiac muscle GLUT4 translocation may represent the differences in severity between the two tissues.

Although we did not co-stain for GLUT-4 and fiber type, it should be noted that most dystrophin-deficient myofibers undergo fiber type switching from fast to slow twitch [4]. We can then infer that GRMD myofibers with GLUT-4 membranous expression were of the slow twitch phenotype. A previous study in human muscle showed small, but significant differences in GLUT-4 expression and fiber type; however, the authors concluded that GLUT-4 protein content was related more closely to activity level than fiber type [33].

We recently revealed that the morphologically spared and hypertrophied GRMD CS muscle had a reduction in expression in several glycolytic enzymes, including phosphoglucomutase-1, 6-phosphofructokinase, and glucose-6phosphate isomerase [4]. In the current study, we evaluated at the mRNA level, HK-1, the first enzyme of the glycolytic pathway, and observed an increased expression in GRMD LDE muscle. These differences in glycolytic enzyme expression may be due to muscle specific changes that occur between hypertrophied (CS) and atrophied (LDE) muscle in GRMD and should be further explored. Nevertheless, the increased HK1 is in keeping with our hypothesized compensatory mechanism within dystrophic muscle to rapidly metabolize glucose.

We then hypothesized that increased sarcolemmal GLUT4 could lead to rapid and immediate glucose uptake into skeletal muscle. Indeed, GRMD dogs had rapid BG uptake with a comcomitant insulin peak at 5-min post-dextrose challenge. In contrast, normal/carrier insulin levels peaked at $15 \mathrm{~min}$. This truncated insulin response shows that the apparently rapid glucose uptake was not due to a larger overall insulin level in GRMD. Interestingly, GRMD dogs had higher basal (predextrose injection) BG and insulin levels, suggesting dystrophic muscle is under higher metabolic demand and requires a slightly higher level of BG and insulin levels to compensate. Area under the BG curves did not differ between GRMD and normal/carrier dogs, perhaps because of our failure to sample frequently over the first $15 \mathrm{~min}$ and continue sampling for $3 \mathrm{~h}$ [34]. Had we employed more frequent early sampling, the slope of GRMD curves might have been increased. In addition, because glucose metabolism varies with age, GRMD and normal/carrier dogs should be matched more closely in future studies. Here, we tested the response to insulin-mediated GLUT4 uptake of glucose. Ideally, contraction-mediated GLUT4 uptake should be tested, but performing a treadmill exhaustion protocol in GRMD dogs, as seen in mice,would not be feasible [35].

We further hypothesized that GLUT-4 localization at the dystrophic myofiber membrane would produce a measurable increase in the immediate uptake of $\left[{ }^{18} \mathrm{~F}\right] \mathrm{FDG}$. In order to force rapid and specific uptake into skeletal muscle, we coadministered insulin and $\left[{ }^{18} \mathrm{~F}\right] \mathrm{FDG}$ tracer at the initiation of scanning. Similar to our IV-GTT results, $\left[{ }^{18}\right.$ F]FDG uptake was higher in GRMD vs. normal/carrier dogs at $5 \mathrm{~min}$ post- $\left[{ }^{18} \mathrm{~F}\right] \mathrm{FDG} /$ insulin administration but not at $1 \mathrm{~h}$, consistent with an early, transient response [36]. Most likely, this early transient response to glucose (and a glucose analog) partially compensates for metabolic dysregulation in dystrophic muscle. Other methods such as dynamic PET after $\left[{ }^{18} \mathrm{~F}\right] \mathrm{FDG}$ administration might demonstrate differences in uptake over time [37]. With regards to exercise, all dogs are provided daily enrichment, including exercise (walking and running outside for a period of time). Normal animals are inherently more active than GRMD dogs, but regular conditioning would be expected to create increased insulin sensitivity in normal muscle (i.e., increasing glucose and glucose analog uptake). For these imaging studies, exercise was minimized in all groups the morning prior to the $\mathrm{PET} / \mathrm{CT}$ studies to reduce any exercise-related short-term effects on $\left[{ }^{18} \mathrm{~F}\right]$ FDG uptake. Nevertheless, our findings in GRMD dogs that increased $\left[{ }^{18} \mathrm{~F}\right] \mathrm{FDG}$ and dextrose uptake at $5 \mathrm{~min}$ postadministration provides a potential biomarker "window" to assess treatments intended to improve muscle metabolism.

Like skeletal muscle, GLUT4 is the major transporter of glucose into cardiomyocytes [38, 39]. Surprisingly, mean and max SUVs were lower on PET studies of the left ventricle in GRMD vs. normal/carrier dogs. Since this reduction persisted beyond the 5-min time point, this likely did not occur simply because of selective skeletal muscle uptake. Instead, this presumably reflects a primary cardiac insulin resistance associated with dystrophic cardiomyopathy [16]. While we further hypothesized that $\left[{ }^{18} \mathrm{~F}\right] \mathrm{FDG}$ distribution would correspond to the regional nature of lesions within the dystrophic heart, mean or max SUV did not vary among the $16 \mathrm{LV}$ segments nor did CoV differ between genotypes. Similarly, GLUT4 protein expression and translocation differences could not be confirmed in the heart, though there was a trend toward increased GLUT4 protein expression in GRMD, which appeared to be affected by higher levels in older dogs. Indeed, further studies in GRMD heart muscle are needed to confirm GLUT-4 trafficking abnormalities due to potential aberrant insulin and/or contraction stimulation.

Taken together, these findings reiterate that glucose dynamics and metabolism vary between cardiac and skeletal muscle. Indeed, these two muscle cell types have different GLUT4 vesicle populations with varying responses to insulin and contraction $[38,39]$. This metabolic dissimilarity could also help explain the difference in disease progression between these two tissues, as suggested by recent gene microarray studies in GRMD dogs [40]. Most affected dogs from the PET/CT study are thriving in the colony, which has precluded assessment of GLUT4 expression in their hearts to better clarify these differences.

Blood flow and inflammation can be contributors to differences in $\left[{ }^{18} \mathrm{~F}\right] \mathrm{FDG}$ uptake, particularly in early PET scans. Due to prolonged washout times and concerns about prolonged anesthesia in the dogs, as well as not wanting to administer an additional tracer to confound results, we did not assess blood flow or inflammation directly in this study. However, blood pressure, which is measured in the pelvic limb, was not significantly different between groups before, during, or after 
$\left[{ }^{18} \mathrm{~F}\right] \mathrm{FDG}$ and insulin administration. Additionally, SUV measurements of pelvic fat, which should not be affected by the GLUT4 differences observed in the skeletal muscle between genotypes, did not show significant differences in mean SUV. However, pelvic fat max SUV was higher in normal dogs compared with other groups. Overall, these results suggest that altered blood flow was not a major contributor to increased skeletal muscle SUV in GRMD dogs.

Likewise, we assessed multiple GRMD muscles in the thigh which naturally show differences in degree of pathology, including inflammation. These include the CS, which is hypertrophied but has minimal inflammation, and the VL and rectus femoris that show classic dystrophic changes of inflammation/degeneration [20,32]. Our statistical analysis revealed that GRMD skeletal muscle had a significant increase in mean SUV at 5 min post- $\left[{ }^{18} \mathrm{~F}\right] \mathrm{FDG} /$ insulin, but the GRMD genotype did not influence uptake between the individual muscles (CS, VL, rectus femoris). Elevated blood glucose from feeding has been shown to interfere with $\left[{ }^{18} \mathrm{~F}\right] \mathrm{DG}$ uptake, while co-administration of insulin results in a rapid uptake of $85-90 \%$ of glucose within the first $5 \mathrm{~min}$ [41]. Additionally, although inflammatory cells have insulin responsive GLUT4 translocation [42], the inflammatory infiltrate present in GRMD muscle would be expected to interfere with insulin sensitivity, rather than increasing uptake [43]. Therefore, we hypothesize that the primary driver of increased SUV in GRMD skeletal muscle was due to insulin-stimulated GLUT-4 uptake of $\left[{ }^{18} \mathrm{~F}\right] \mathrm{DG}$. We acknowledge that further studies should be performed to co-administer $\left[{ }^{18} \mathrm{~F}\right] \mathrm{FDG}$, a blood flow tracer, and an inflammatory marker in GRMD dogs to further clarify this issue.

Another potential confounding factor is the age differences between the molecular and PET studies. For our initial molecular assessment of mRNA, protein expression, and qPCR, we used a cohort of frozen samples banked from previous biopsies and muscle sampling at disease-specific time points. When we moved to in vivo imaging, we utilized breeder dogs with a wider age range that were currently available and attempted to best match between the examined groups. It is possible that the gene expression and GLUT4 localization profiles in older, affected dogs are different than those seen in younger animals. However, disease differences are manifested and stratified by 6 months of age, and would be expected to be similar or worse in older affected dogs.

Gender is also a potential confounding factor. In people, there are differences in GLUT4 expression and insulin resistance between males and females [41]. Carrier animals in this study were female, while GRMD and normal animals were of mixed gender. Female dogs in diestrus or pregnancy can have increased insulin resistance and higher insulin, but we did not include females which had recently been in heat in this study. However, analysis of glucose or insulin levels stratified by gender did not show a significant difference between genders in our cohort. Previous canine studies of circulating monocyte glucose transporters did not show a difference between gender [42]. We have not detected any functional differences between genders in GRMD dogs, either [44]. As such, we believe sex differences were not a major confounder in this study.

\section{Conclusions}

Overall, our results suggest that glucose handling and metabolism differs between GRMD and normal/carrier dogs. In particular, GRMD dogs have higher levels of both resting insulin and glucose, more rapid glucose uptake, which peaked at different levels compared to normal dogs. We theorize that increased GLUT4 at the cell membrane primes GRMD muscle to induce rapid glucose uptake. These data suggest that glucose metabolism, specifically GTT and PET/ CT testing, may be utilized as surrogate biomarkers to assess disease progression and normalization of muscle metabolism in GRMD dogs following various treatments. Further studies are needed to determine if GRMD cardiac cells are more or less insulin responsive than normal. Future imaging studies to evaluate tracers of inflammation, blood flow and fatty acid metabolism in the heart/skeletal muscle would further resolve some of the remaining questions regarding confounding factors of $\left[{ }^{18} \mathrm{~F}\right] \mathrm{FDG}$ uptake in GRMD.

Acknowledgements. We would like acknoledge Jeff Morin for his discussions. The use of the Microscopy and Imaging Center facility at Texas A\&M University is acknowledged.

Funding Information This study was supported by a startup package from Texas A\&M University (PPN). Dr. Schneider's salary was supported by Zoetis-Morris Animal Foundation Fellowship D14CA903. The Olympus FV1000 confocal microscope acquisition was supported by the Office of the Vice President for Research at Texas A\&M University.

Compliance with Ethical Standards

\section{Conflict of Interest Statement}

Dr. Kornegay reports personal fees from Solid Biosciences, Cambridge, MA, outside the submitted work. The other authors declare that they have no conflict of interest.

\section{Ethical Approval}

All applicable institutional and/or national guidelines for the care and use of animals were followed.

Open Access This article is distributed under the terms of the Creative Commons Attribution 4.0 International License (http:// creativecommons.org/licenses/by/4.0/), which permits unrestricted use, distribution, and reproduction in any medium, provided you give appropriate credit to the original author(s) and the source, provide a link to the Creative Commons license, and indicate if changes were made.

\section{References}

1. Mendell JR, Shilling C, Leslie ND et al (2012) Evidence-based path to newborn screening for Duchenne muscular dystrophy. Ann Neurol 71:304-313

2. Hoffman E, Brown R, Kunkel L (1987) Dystrophin: the protein product of the Duchenne muscular dystrophy locus. Cell 51:919-928 
3. McDonald C, Abresch R, Carter G et al (1995) Profiles of neuromuscular diseases: Duchenne muscular dystrophy. Am J Phys Med Rehabil 995:S70-S92

4. Nghiem P, Bello L, Stoughton W et al (2017) Changes in muscle metabolism are associated with phenotypic variation in golden retriever muscular dystrophy. Yale J Biology Med 90:351-360

5. Timpani C, Hayes A, Rybalka E (2015) Revisiting the dystrophinATP connection: how half a century of research still implicates mitochondrial dysfunction in Duchenne muscular dystrophy aetiology. Med Hypotheses 85:1021-1033

6. Onopiuk B, Wierzbicka K et al (2009) Mutation in dystrophinencoding gene affects energy metabolism in mouse myoblasts. Biochem Bioph Res Comm 386:463-466

7. Sharma U, Atri S, Sharma M et al (2003) Skeletal muscle metabolism in Duchenne muscular dystrophy (DMD): an in-vitro proton NMR spectroscopy study. Magn Reson Imaging 21:145-153

8. Chi H, McKee D et al (1987) Effect of Duchenne muscular dystrophy on enzymes of energy metabolism in individual muscle fibers. Metabolism 36:761-767

9. Rando T (2002) Oxidative stress and the pathogenesis of muscular dystrophies. Am J Phys Med Rehabil 81:S175-S186

10. Birnbaum (1989) Identification of a novel gene encoding an insulinresponsive glucose transporter protein. Cell 57:305-315

11. Rodríguez-Cruz M, Sanchez R, Escobar R et al (2015) Evidence of insulin resistance and other metabolic alterations in boys with Duchenne or Becker muscular dystrophy. Int J Endocrinol 2015:1-8

12. Olichon-Berthe C, Gautier N, Obberghen E, Marchand-Brustel Y (1993) Expression of the glucose transporter GLUT4 in the muscular dystrophic mdx mouse. Biochem J 291:257-261

13. Klip A, Sun Y, Chiu T, Foley K (2014) Signal transduction meets vesicle traffic: the software and hardware of GLUT4 translocation. Am J Physiol Cell Physiol 306:C879-C886

14. Lund S, Holman GD, Schmitz O, Pedersen O (1995) Contraction stimulates translocation of glucose transporter GLUT4 in skeletal muscle through a mechanism distinct from that of insulin. Proc National Acad Sci (USA) 92:5817-5821

15. Luiken JJ, Glatz JF, Neumann D (2015) Cardiac contraction-induced GLUT4 translocation requires dual signaling input. Trends Endocrinol Metabolism 26:404-410

16. Nikolaidis LA, Sturzu A, Stolarski C et al (2004) The development of myocardial insulin resistance in conscious dogs with advanced dilated cardiomyopathy. Cardiovasc Res 61:297-306

17. Kornegay JN, Tuler SM, Miller DM, Levesque DC (1988) Muscular dystrophy in a litter of golden retriever dogs. Muscle Nerve 11:10561064

18. Sharp NJ, Kornegay JN, Van Camp SD et al (1992) An error in dystrophin mRNA processing in golden retriever muscular dystrophy, an animal homologue of Duchenne muscular dystrophy. Genomics 13:115-121

19. Terrill J, Duong M, Turner R et al (2016) Levels of inflammation and oxidative stress, and a role for taurine in dystropathology of the golden retriever muscular dystrophy dog model for Duchenne muscular dystrophy. Redox Biol 9:276-286

20. Nghiem P, Hoffman E, Mittal P et al (2013) Sparing of the dystrophin-deficient cranial sartorius muscle is associated with classical and novel hypertrophy pathways in GRMD dogs. Am J Pathology 183:1411-1424

21. Kornegay J, Spurney C, Nghiem P et al (2014) Pharmacologic management of Duchenne muscular dystrophy: target identification and preclinical trials. ILAR J 55:119-149

22. Momose M, Iguchi N, Imamura K et al (2001) Depressed myocardial fatty acid metabolism in patients with muscular dystrophy. Neuromuscul Disord 11:464-469

23. Nishimura T, Yanagisawa A, Sakata H, Sakata K, Shimoyama K, Ishihara T, Yoshino H, Ishikawa K (2001) Thallium-201 single photon emission computed tomography (SPECT) in patients with Duchenne's progressive muscular dystrophy. Jpn Circulation J 65:99105

24. Perloff JK, Henze E, Schelbert HR (1984) Alterations in regional myocardial metabolism, perfusion, and wall motion in Duchenne muscular dystrophy studied by radionuclide imaging. Circulation 69:33-42
25. Santacatterina F, Chamorro M, Arenas CN et al (2015) Quantitative analysis of proteins of metabolism by reverse phase protein microarrays identifies potential biomarkers of rare neuromuscular diseases. J Transl Med 13:1-11

26. Rayavarapu S, Coley W, Cakir E, Jahnke V, Takeda S, Aoki Y, Grodish-Dressman H, Jaiswal JK, Hoffman EP, Brown KJ, Hathout Y, Nagaraju K (2013) Identification of disease specific pathways using in vivo SILAC proteomics in dystrophin deficient $\mathrm{mdx}$ mouse. Mol Cell Proteomics 12:1061-1073

27. Kemp GJ, Taylor DJ, Dunn SP et al (1993) Cellular energetics of dystrophic muscle. J Neurol Sci 116:201-206

28. Chen Y-W, Zhao P, Borup R, Hoffman E (2000) Expression profiling in the muscular dystrophies: identification of novel aspects of molecular pathophysiology. J Cell Biol 151:1321-1336

29. Richter E, Hargreaves M (2013) Exercise, GLUT4, and skeletal muscle glucose uptake. Physiol Rev 93:993-1017

30. Garvey WT, Maianu L, Hancock JA et al (1992) Gene expression of GLUT4 in skeletal muscle from insulin-resistant patients with obesity, IGT, GDM, and NIDDM. Diabetes 41:465-475

31. Garvey WT, Maianu L, Zhu JH et al (1998) Evidence for defects in the trafficking and translocation of GLUT4 glucose transporters in skeletal muscle as a cause of human insulin resistance. J Clin Invest 101:2377-2386

32. Kornegay JN, Cundiff DD, Bogan JR et al (2003) The cranial sartorius muscle undergoes true hypertrophy in dogs with golden retriever muscular dystrophy. Neuromuscul Disord 13:493-500

33. Daugaard JR, Nielsen JN, Kristiansen S, Andersen JL, Hargreaves M, Richter EA (2000) Fiber type-specific expression of GLUT4 in human skeletal muscle: influence of exercise training. Diabetes 49:10921095

34. Pacini G, Bergman R (1986) MINMOD: a computer program to calculate insulin sensitivity and pancreatic responsivity from the frequently sampled intravenous glucose tolerance test. Comput Meth Prog Bio 23:113-122

35. Howlett KF, Andrikopoulos S, Proietto J, Hargreaves M (2013) Exercise-induced muscle glucose uptake in mice with graded, musclespecific GLUT-4 deletion. Physiol Rep 1:e00065

36. Ahmad N, Welch I, Grange R et al (2011) Use of imaging biomarkers to assess perfusion and glucose metabolism in the skeletal muscle of dystrophic mice. BMC Musculoskel Dis 12, 12(1)

37. Reinhardt N, Beu M, Vosberg H et al (1999) Quantification of glucose transport and phosphorylation in human skeletal muscle using FDG PET. J Nucl Med 40:977-985

38. Zorzano A, Sevilla L, Tomàs E et al (1997) GLUT4 trafficking in cardiac and skeletal muscle: isolation and characterization of distinct intracellular GLUT4-containing vesicle populations. Biochem Soc T 25:968-974

39. Horie T, Ono K, Nagao K et al (2008) Oxidative stress induces GLUT4 translocation by activation of PI3-K/Akt and dual AMPK kinase in cardiac myocytes. J Cell Physiol 215:733-742

40. Markham LW, Brinkmeyer-Langford CL, Soslow JH et al (2017) GRMD cardiac and skeletal muscle metabolism gene profiles are distinct. BMC Med Genet 10:21

41. DeFronzo RA, Gunnarsson R, Björkman O et al (1985) Effects of insulin on peripheral and splanchnic glucose metabolism in noninsulin-dependent (type II)diabetes mellitus. J Clin Invest 76:149-155

42. Schnurr TM, Reynolds AJ, Gustafson SJ et al (2014) Conditioning causes an increase in glucose transporter-4 levels in mononuclear cells in sled dogs. Int J Biochem Cell Biology 55:227-231

43. Varma V, Yao-Borengasser A, Rasouli N, Nolen GT, Phanavanh B, Starks T, Gurley C, Simpson P, McGehee RE Jr, Kern PA, Peterson CA (2009) Muscle inflammatory response and insulin resistance: synergistic interaction between macrophages and fatty acids leads to impaired insulin action. Am J Physiol Endocrinol Metab 296:E1300E1310

44. Kornegay JN, Bogan JR, Bogan DJ et al (2011) Golden retriever muscular dystrophy (GRMD): developing and maintaining a colony and physiological functional measurements. Methods Mol Biol 709:105-123 